\title{
Ungueal Psoriasis: A Single Nail Affected
}

\section{Potin ME*, Rocha C, Sehtman A and Allevato MA}

Dermatology Division, Hospital de Clínicas “José de San Martín”, Argentina

*Corresponding author: María Eugenia Potin, Dermatology Division. Hospital de Clínicas "José de San Martín"- Córdoba 2351 Street (CABA-Buenos Aires-Argentina), Tel: +54-0221-15-4389400; Email: eugeniapotin@hotmail.com

\section{Case Report \\ Volume 2 Issue 1}

Received Date: January 16, 2017

Published Date: February 07, 2017

DOI: $10.23880 /$ cdoaj16000113

\section{Abstract}

Nail involvement is an extremely common feature of psoriasis and affects approximately $10-78 \%$ of psoriasis patients, while isolated nail psoriasis occurs in $5-10 \%$ of patients.

We communicate a patient whose case represented some difficulties in the diagnosis and treatment, but rich of teaching.

Keywords: Psoriasis; Nail involvement

\section{Case Report}

A 27-year-old female Colombian patient, who lives and attend college in our country were presented at our onycopathology unit for a nail injury on the fifth finger of her right hand of 6 months of evolution with occasionally painful, for which she had not received previous treatments. She had no personal or family history of skin disorders.

Dermatological examination revealed in the nail plate on the right little finger: onydystrophy, onycholysis, oil stain with erythematous border without any other nail or skin involvement (Figure 1).

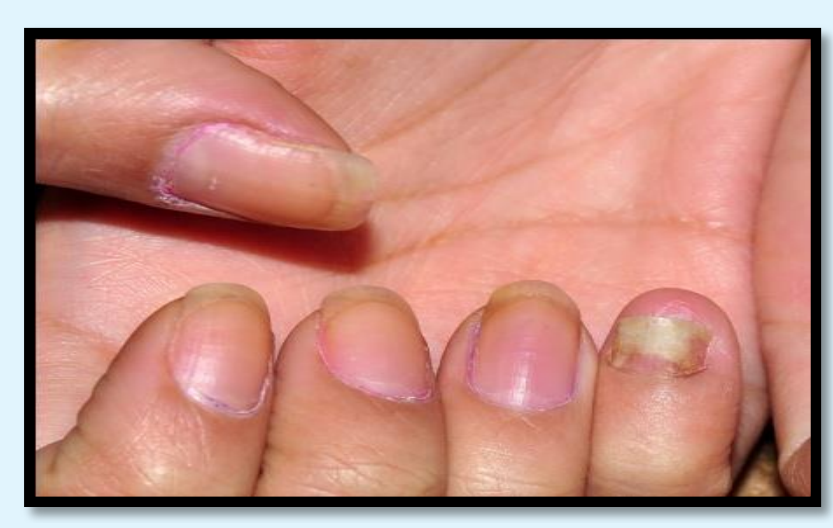

Figure 1: Nail plate on the right little finger
In the dermoscopy examination we found vasodilatation of the bed vessels, without other findings. Also, mycological studies (direct and cultures) were performed with negative results. A chest $\mathrm{x}$ ray showed no abnormalities and a blood lab test informed: RBC, WBC, platelets, hemoglobin, hematocrit and hepatogram within normal values.

A biopsy of the nail bed was considered. The anatomopathological report showed the presence of tortuous vasodilation of capillaries (Figure 2) and neutrophil microabscesses (Figure 3).

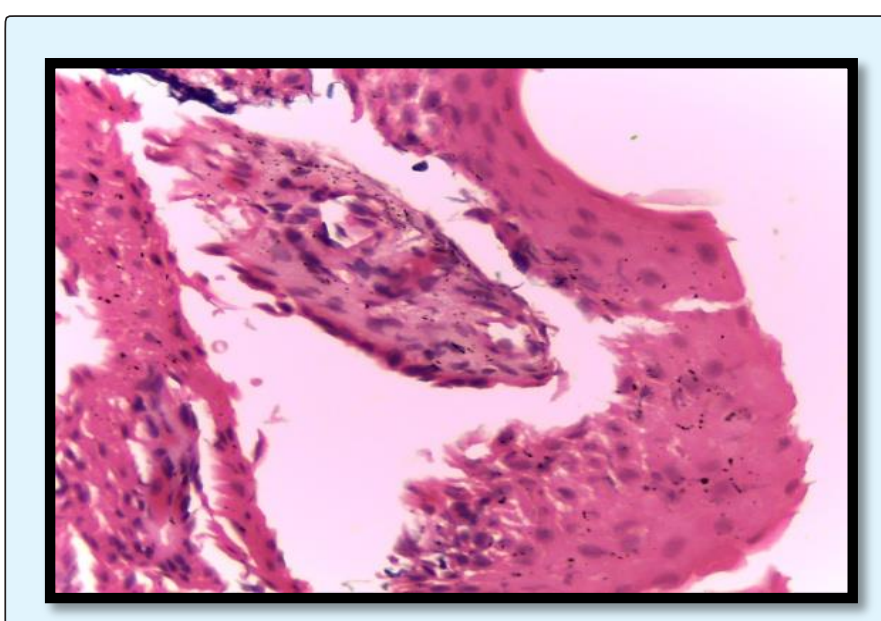

Figure 2: Presence of tortuous vasodilation of capillaries 


\section{Clinical Dermatology Open Access Journal}

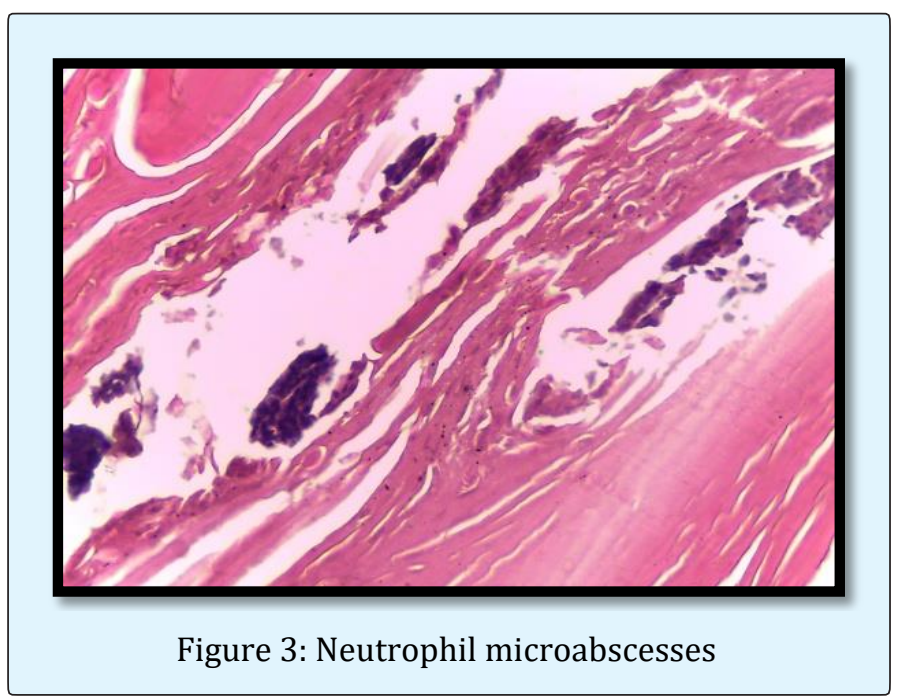

Our final diagnosis was: single nail psoriasis.

With the clinic of extensive involvement of a single nail plate and discarding arthropathetic and cutaneous pathology, we decided to start treatment with infiltrations with $0.5 \mathrm{cc}$ triamcinolone acetonide every 15 days, with a total of 5 applications (Figure 4).

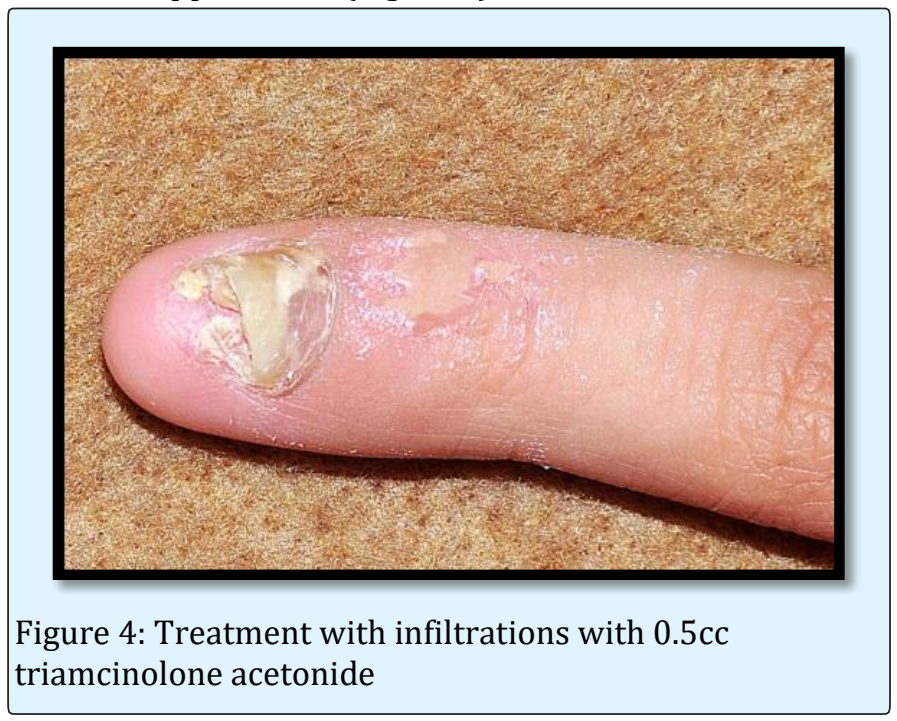

We did not get an adequate response to this treatment and subsequently with the worsening of onycholysis and onychodystrophy, we finally decided the excision of the nail plate.

Two months later, for our pleasant surprise, we found a suitable regrowth of the nail plate on the fifth finger of her right hand; around a 50\% growth of healthy nail (Figure $5)$.

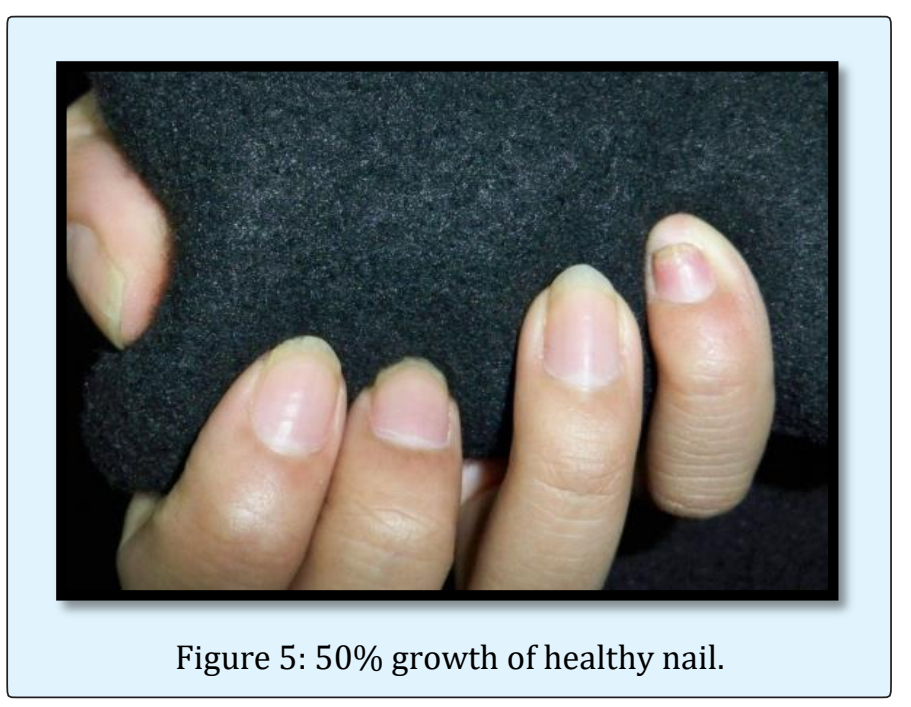

\section{Discussion}

Isolated ungueal psoriasis is a rare entity and accounts for only $5-10 \%$ of nail psoriasis. Nail involvement is an extremely common feature of psoriasis and affects approximately $10-78 \%$ of psoriasis [1].

When nail bed involvement occurs, clinical signs such as oil spots, onycholysis, subungual hyperkeratosis, and splinter haemorrhages may be observed [2].

Nail psoriasis engenders both physical and psychological handicap, leading to significant negative repercussions in the quality of life [3].

Diagnosis of nail psoriasis can be made easily in a patient with concomitant skin psoriasis. However, in cases of isolated nail psoriasis, this can be presenting with a diagnostic dilemma to a dermatologist, for this reason the dermoscopy and a nail biopsy can be used [4].

Nail disease is usually overlooked in the management of psoriasis, with skin involvement being the primary concern. Also, treatment of nail psoriasis is a big challenge for a dermatologist for some many reasons like: the poor drug delivery, a long duration of treatment required because of the slow rate of nail growth and very important the faults in the diagnosis [5].

In the literature, multiple treatments have been proposed, with topical and intralesional corticosteroids being the first line of choice [6], as it was done in the case of our patient who had infiltrations with triamcinolone but with a few response, probably due to the extensive involvement of the nail bed. 


\section{Clinical Dermatology Open Access Journal}

It is striking that when performing excision of the nail plate, the posterior growth was normal without requiring other treatments.

What we want to share with the presentation of this clinical case is that we believe that the application of topical corticosteroids generated an anti-inflammatory effect on the nail bed producing a polymorphonuclear infiltrate which, later, with the nail excision allowed adequate nail plate growth. We consider that in severe cases where the diagnosis of ungueal psoriasis with only nail bed involvement and with severe onycholysis is determined, ungueal excision after cortico-therapy through infiltrations may represent a good therapeutic possibility.

\section{References}

1. Dogra A, Arora AK (2014) Nail Psoriasis: The Journey so far. Indian J Dermatol 59 (4): 319-333.

2. Sánchez-Regaña $M$, Umberger $P$ (2008) Diagnostic and therapeutic aspects of nail psoriasis. Actas Dermosifiliogr 99(1): 34-43.
3. De Jong EM, Seegers BA, Gulinck MK, Boezeman JB, van de Kerkhof PC (1996) Psoriasis of the nails associated with disability in a large number of patients: results of a recent interview with 1728 patients. Dermatology 193(4): 300-303.

4. Barreiro Pérez L, Lopez Lopez R, Gonzalez Ramos J (2014) Psoriasis ungueal. An Pediatr (Barc) 81(6): e14-e15.

5. De Vries AC, Bogaards NA, Hooft L, Velema M, Pasch $M$, et al. (2013) Interventions for nail psoriasis. Cochrane Database Syst Rev 1: CD007633.

6. Cantoresi F, Sorgi P, Arcese A, Bidoli A, Bruni F, et al. (2009) Improvement of psoriatic onychodystrophy by a water-soluble nail lacquer. J Eur Acad Dermatol Venereol 23 (7): 832-834.

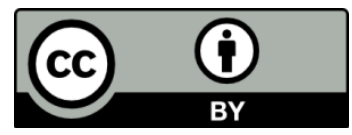

